\title{
Manifestaciones de Conducta Disruptiva y Comportamiento Perturbador en Población Normal de 4 A 17 Años de Edad.
}

Manifestations of Disruptive Behavior and Perturbation

Behavior in Normal Populations of 4 and 17 Years of Age.
Recibido: Enero 2008 revisado: Junio 2008
Por: Grace Arias Correa, Eliana Montoya Roldán, Maria Gladys Romero.

\section{Resumen:}

El presente articulo presenta resultados de investigación el cual tenía como objetivo identificar casos con alta probabilidad de manifestar conductas externalizantes en la población normal, para ello se realizó una investigación de corte transversal con una muestra de 444 participantes a los cuales se aplicó el instrumento SNAP-IV (Swanson, Nolan \& Pelham, 1983). Los resultados sugieren: diferencias en la información suministrada por cada una de las fuentes (padres y maestros) y la presencia de conductas con alta probabilidad de riesgo que pueden ser intervenidas en el medio escolar y familiar.

\section{Palabras Claves:}

Conducta disruptiva, comportamiento perturbador, autorregulación, desarrollo moral.

\section{Abstract:}

This current paper shows the results of a research whose aim was to identify highprobability cases showing externalizing behaviors in the normal population, a crosssection study was designed with a sample of 444 participants, who were given the SNAPIV (Swanson, Nolan \& Pelham, 1983). The results showed differences in the Information provided by each one of the sources (parents and teachers) as well as the presence of behaviors with high-risk probability, which may be intervened in the school or family setting.

\section{Key words:}

Disruptive behavior, perturbation behavior, self-regulation, moral development. 


\section{Introducción}

El ajuste comportamental y social se ve influenciado por dos tipos de conductas: las conductas internalizantes que se refieren a la ansiedad y depresión como fuentes de sentimientos de soledad, temor, inferioridad, culpa, tristeza y desconfianza; y las conductas externalizantes que se refieren directamente a las conductas que afectan a otros tales como: rompimiento de normas, robo, fraude y mentira, conflictos frecuentes con una ausencia de sentimientos de culpa y rechazo a la aceptación de la autoridad y las normas sociales (Achenbah \& Rescola, 2001).

Los trastornos internalizados no son fáciles de valorar o identificar. Son comportamientos perturbadores para el niño y el adolescente, pero usualmente no son tan evidentes para los padres y el maestro. Estos problemas se refieren a comportamientos de ajuste ambiental que se observan como conductas de inhibición, timidez, tristeza y dificultades para la expresión emocional. Se incluyen dentro de este tipo de trastornos, la depresión, la ansiedad y la somatización. En términos generales la depresión, se caracteriza por un estado de ánimo disfórico, con presencia de sentimientos de infelicidad, ideas de suicidio, aislamiento y autorreproches frecuentes. Por su parte, la ansiedad es un mecanismo que consiste en una tendencia nerviosa de preocupación por problemas reales o imaginarios; son frecuentes las manifestaciones de miedos, fobias y conductas de desaprobación. Secundario a estas manifestaciones también se pueden presentar somatización (Reynold \& Kamphaus, 1992).

Según la Asociación Americana de Psiquiatría, (2002) en el manual de los trastornos mentales (DSM-IV-TR), dentro de los "Trastornos del estado de ánimo" se encuentra la depresión comúnmente relacionada con la tristeza. Por otro lado el manual considera los "Trastornos de ansiedad" referidas a sensaciones angustiosas dentro de las cuales se encuentran entre otros la ansiedad generalizada. Por su parte, la ansiedad y la depresión constituyen los dos motivos de consulta, más frecuentes de la psicología y la psiquiatría.

Por el contrario, los trastornos externalizados son muy evidentes para el contexto ambiental (es decir, padres, maestros y compañeros). En la infancia y la adolescencia se caracterizan por excesos conductuales o perturbaciones evidentes. En la conducta infantil se refieren a las alteraciones del control del comportamiento tales como la autorregulación, la actividad motora, el control de las emociones y las habilidades de juicio social; estos comportamientos perturbadores se observan como agresividad que es la tendencia a actuar hostilmente o con actitud amenazante hacia otros de forma verbal o física; en la hiperactividad hay una tendencia a ser excesivamente activo, con ejecuciones impulsivas y precipitadas. Finalmente, en los problemas de conducta se identifican por un comportamiento que muestra insensibilidad ante el 
dolor o las necesidades de los otros y una clara dificultad para el cumplimiento de normas y reglas que pueden incluir el daño a la propiedad de otros (Reynold \& Kamphaus, 1992).

Los problemas externalizantes constituyen unas de las características más observables del Trastorno por Déficit de Atención (TDAH), El Trastorno Oposicionista Desafiante (TOD) y el Trastorno Disocial de la Conducta (TDC). Es así como en el TDAH las manifestaciones de hiperactividad, impulsividad, dificultad para permanecer en el puesto y la tarea son problemas de conductas externalizantes claramente observables en este trastorno (Sattler, 2000).

Colombia en la actualidad es uno de los países más violentos, como producto de la historia política del país y el fenómeno específico del narcotráfico, que hace de la violencia y la violación de las normas una manifestación cíclica que acercan a niños y adolescentes a realidades y experiencias de comportamientos violentos latentes y manifiestos (Cano \& Castillo, 2002).

Los problemas de conducta, incluyendo comportamiento negativista, desafíos, agresiones y delincuencia, constituyen la mayoría de la comorbilidad hallada en niños con TDAH. Los síntomas de trastorno negativista desafiante incluyen desafío a la autoridad, no cumplir las demandas de los adultos, intimidar, culpar a otros y presentar otras conductas que constituyen transgresiones menores de normas sociales apropiadas a su edad. En cambio el trastorno disocial describe a niños con conductas agresivas y delictivas que representan transgresiones importantes de normas sociales apropiadas a la edad. Los síntomas del trastorno disocial pueden ser tanto agresivos (p.ej., iniciar peleas, llevar armas o cometer asaltos) como no agresivos (p. ej., mentir, hacer novillos, escaparse de casa o hurtar) (Newcorn \& Halperin, 2003; como cita en Brown, 2003).

Kholberg (1987), basado en la perspectiva piagetiana, describió seis etapas del desarrollo moral en tres niveles generales que fueron definidas según la estructura de la inteligencia. Estas etapas consideradas como invariables y universales, explican las secuencias de pasos que las personas atraviesan en un orden fijo y que permiten un concepto escalonado de justicia más consciente y moralmente adecuado. En el nivel preconvencional, la moralidad está controlada externamente. En el nivel post- convencional se mueven más allá del apoyo incuestionable de las reglas y leyes de su propia sociedad, es una moral regida de manera interna (Colby \& Kholberg, 1987).

Teóricamente dos factores de riesgo se han relacionado con las conductas externalizantes: las diferencias individuales en la capacidad de los niños para regular la conducta moral y las variaciones en la práctica de la disciplina paterna y escolar. En el caso del oposicionismo 
desafiante (caracterizado por una activa omisión de las normas y peticiones) estaría relacionado con un insuficiente desarrollo moral. Esto porque la internalización y el desarrollo de la norma reflejarían desde esta perspectiva teórica, la autorregulación que se expresa en la capacidad para evaluar la conducta inapropiada, la posibilidad de restringir esta conducta evaluada como mala y la tendencia a corregir los daños a través de la confesión y la reparación. En otra perspectiva, los estudios que han relacionado si el desarrollo moral explica las conductas externalizantes no han mostrado la correlación esperada y las perspectivas neurobiológicas para la comprensión de las sicopatías han relacionado estas conductas perturbadoras con escasa sensibilidad moral (Kerr, López, Olson \& Sameroff, 2004; Ostrosky, 2008).

En un estudio de caso de dos pacientes con daño de corteza pre frontal a partir del estudio de la historia clínica y de neuroimagen cerebral, en el análisis retrospectivo de los casos, sus profesores observaban que estas personas "nunca mostraban sentimientos de culpa o remordimiento por sus actos". No se encontró en ellos ninguna evidencia de empatía, culpaban a otros de sus dificultades sociales y eran incapaces de tomar decisiones o evaluar las consecuencias de sus actos. La lesión en la corteza pre frontal, sobre todo si se da a edades muy tempranas, tiene como consecuencia un desarrollo anormal de la conducta moral. Estas personas mostraron, por otro lado, capacidades intelectuales normales como, por ejemplo, aritmética mental, razonamiento verbal, percepción visoespacial (Damasio, et al., 1999; Anderson, et al., 1999).

La autorregulación se entiende como las construcciones de la modulación de la atención visual, el control efectivo de la capacidad de suprimir un comportamiento dominante con el fin de realizar uno subdominante y por último el automonitoreo. La autorregulación permite un desempeño exitoso en las actividades de orden cognitivo y comportamental. La mejor comprensión de los factores que contribuyen al desarrollo de la autorregulación se relacionan con los procesos individuales (i,e la cognición y la atención) y extraprocesos individuales (i,e estilos de crianza, practicas escolares de la disciplina) (Kopp \& Wyer, 1994; Kochanska et al., 2000; Rothbart \& Bates, 1998; Demetriou, 2000; como cita en Jennings et al, 2008).

\section{Método e instrumentos:}

Con el objetivo de identificar casos con alta probabilidad de manifestar trastornos externalizantes en la población normal evaluada, se diseñó un estudio de corte transversal. La muestra aleatoria por método de sorteo incluyó colegios públicos y privados del área metropolitana de Medellín, previa aceptación de participación en el estudio a través del consentimiento informado; los padres y maestros reportaron en los cuestionarios SNAP-IV la frecuencia de conductas externalizantes. 
Con una muestra final de 444 participantes vinculados al estudio, se dividieron en tres grupos: un primer grupo conformado por niños(as) de 4 a 7 años de edad; un segundo grupo conformado por niños(as) de 8 a 12 años y un grupo de jóvenes con una edad entre los 13 y 17 años. La información se procesa y analiza utilizando los programas ACCES, Excel 4.0 y SPSS 15.0 .

Se utilizó para el estudio la escala SNAP-IV de Swanson, Nolan y Pelham (SNAP) (Swanson et al, 1983). Este es un cuestionario con reactivos basados en los criterios del DSM-IV de utilidad en una gama amplia de trastornos infantiles entre los cuales el Trastorno por déficit de atención (TDAH) se encuentra incluido en dos subtest de síntomas: Inatención (ĺtems 1-9) e Hiperactividad-Impulsividad (ítems 11-19). También posee ítems de los criterios del DSM-IV para Trastorno oposicionista desafiante (TOD), (ítems 21-28), que generalmente se pueden presentar en los niños con TDAH. Además de ítems basados en el DSM-IV para TDAH y TOD, contiene ítems de la escala de Conners (Conners, 1979) y de la Escala de Conners de IOWA (Loney \& Milich, 1985).

EI SNAP-IV para padres y maestros, versión en español aprobada por el autor esta basado en una escala de calificación tipo likert de 0 a 3, que corresponden a 4 respuestas: Nunca=0; A veces $=1$; frecuentemente $=2$ y siempre $=3$. Las puntuaciones de las Sub-Escalas son calculadas al sumar los puntajes obtenidos en los ítems que se encuentran subdivididos de acuerdo a diferentes trastornos.

Finalmente el SNAP-IV incluye los 10 últimos ítems de la Escala de Swanson, Kotkin, Agler, Mylnn, and Pelham (SKAMP). Estos ítems son manifestaciones en el aula de clases para inatención, hiperactividad e impulsividad. Estos se pueden usar para estimar la severidad de problemas en el colegio. Es importante tener en cuenta que muchos trastornos pueden producir alteraciones en el aula de clases, que no necesariamente se deban a un TDAH.

En el cuadro 1 se encuentran los ítems del SNAP-IV analizados en este estudio, con los rangos de valores posibles para cada ítem y el puntaje máximo directo para cada una de las conductas evaluadas. 


\section{Cuadro 1}

Reactivos y puntuaciones máximas para conductas externalizantes, del cuestionario SNAP-IV

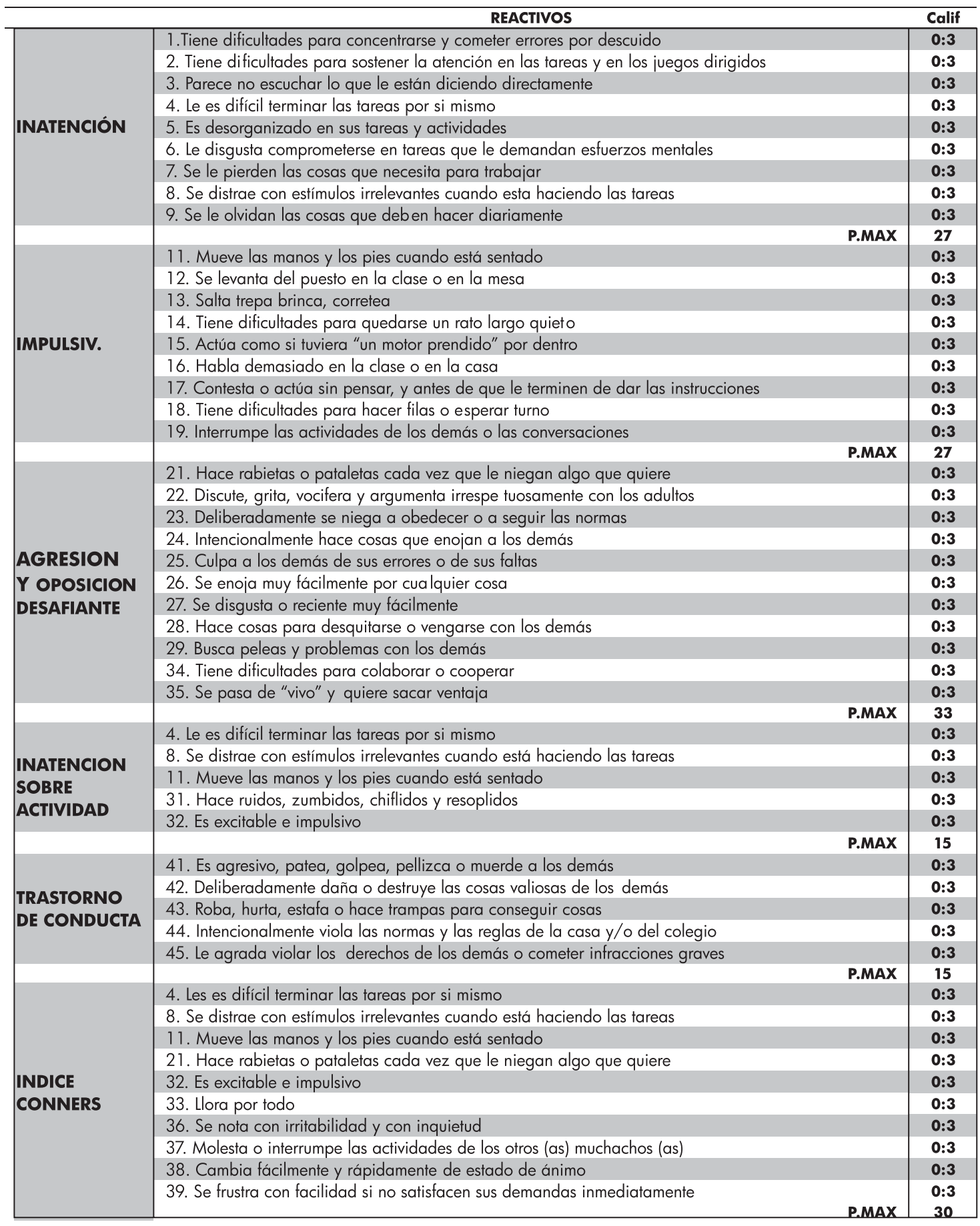




\section{Resultados}

Si bien la selección de la muestra fue aleatoria, hay mayor representatividad del género femenino con una diferencia porcentual del $24.7 \%$ con relación a los hombres. Igualmente la edad de mayor representación está entre los 8 y 12 años de edad; el mayor número de participantes cursaban el ciclo básico de educación (i.e preescolar y primaria). Se vincularon al estudio 5 colegios privados y 7 oficiales. La proporción de estrato bajo tiene mayor representatividad si se compara con los estratos medio y alto. Ver tabla 1.

\section{Tabla 1}

Distribución absoluta y porcentual del grupo de 444 niños y jóvenes evaluados con el SNAP-IV entre 4 y 17 años de edad

\begin{tabular}{|l|c|c|}
\hline & N= 444 & Porcentaje \\
\hline Sexo & 277 & 62.3 \\
\hline Femenino & 167 & 37.6 \\
Masculino & 119 & 26.8 \\
Estrato socioeconómico & 118 & 26.5 \\
Alto & 207 & 46.6 \\
Medio & 53 & 11.9 \\
Bajo & 208 & 46.8 \\
Grupos de edad & 183 & 41.2 \\
De 4 a 7 años & & \\
De 8 a 12 años & 97 & 21.8 \\
De 13 a 14 años & 157 & 35.3 \\
\hline Escolaridad & 190 & 42.7 \\
\hline Preescolar & & \\
Primaria & & \\
Secundaria & & \\
\hline
\end{tabular}

Para el análisis se agruparon los registros con los valores: 2 y 3; equivalentes a las conductas reportadas como: frecuentemente y siempre. Éstas, se denominaron para el estudio como conductas con frecuencias de riesgo, es decir con alta probabilidad de presentar las conductas externalizantes analizadas. 
Los maestros registran un mayor porcentaje de conductas externalizantes que los padres en los diferentes ítems que abarcan tareas de concentración, seguimiento de instrucciones y normas en los rangos de edad de 4 a 7 años. En contraste, las apreciaciones de los padres para las mismas conductas con relación a los maestros, se incrementan a medida que aumenta la edad. Ver Tabla 2.

\section{Tabla 2}

Porcentajes de conductas de Inatención, Impulsividad e Hiperactividad en nivel de riesgo para los diferentes reactivos según el grupo de edad

\begin{tabular}{|c|c|c|c|c|c|c|}
\hline & \multicolumn{2}{|c|}{$\begin{array}{c}4-7 \\
\text { Años de edad } \\
87(100 \%) \\
\end{array}$} & \multicolumn{2}{|c|}{$\begin{array}{c}8-12 \\
\text { Años de edad } \\
202(100 \%) \\
\end{array}$} & \multicolumn{2}{|c|}{$\begin{array}{c}13-17 \\
\text { Años de edad } \\
119(100 \%)\end{array}$} \\
\hline INATENCIÓN & Maestros & Padres & Maestros & Padres & Maestros & Padres \\
\hline Concentración. Errores por descuido & $24 \%$ & $16 \%$ & $32.2 \%$ & $33.1 \%$ & $26,0 \%$ & $35.2 \%$ \\
\hline Sostener la atención en tarea y juego & $24 \%$ & $12.6 \%$ & $21.2 \%$ & $28.7 \%$ & $24.3 \%$ & $21.8 \%$ \\
\hline Escuchar lo que le dicen & $18 \%$ & $11.4 \%$ & $20.7 \%$ & $28.2 \%$ & $23.5 \%$ & $42 \%$ \\
\hline Terminar las tareas por si mismo & $21 \%$ & $11.4 \%$ & $19.3 \%$ & $22.7 \%$ & $21 \%$ & $26 \%$ \\
\hline organización en sus tareas y actividades & $20.6 \%$ & $6.8 \%$ & $22.2 \%$ & $25.7 \%$ & $19.3 \%$ & $20.1 \%$ \\
\hline Compromiso con tareas de esfuerzo mental & $21 \%$ & $12.6 \%$ & $19.3 \%$ & $25.7 \%$ & $21.8 \%$ & $24.3 \%$ \\
\hline Perdida de elementos de trabajo & $17.2 \%$ & $14.9 \%$ & $16.8 \%$ & $33.1 \%$ & $21 \%$ & $30.2 \%$ \\
\hline Distracción con estímulos irrelevantes & $24 \%$ & $13.7 \%$ & $21.7 \%$ & $35.6 \%$ & $25.2 \%$ & $40.3 \%$ \\
\hline Olvido de responsabilidades diarias & $20.6 \%$ & $11.4 \%$ & $22.2 \%$ & $30.1 \%$ & $19.3 \%$ & $26 \%$ \\
\hline \multicolumn{7}{|l|}{ IMPULSIVIDAD } \\
\hline Mover manos y pies al estar sentado & $20.6 \%$ & $14.9 \%$ & $12.8 \%$ & $28.7 \%$ & $3.3 \%$ & $33.6 \%$ \\
\hline Levantarse del puesto & $20.6 \%$ & $16 \%$ & $15.8 \%$ & $24.7 \%$ & $16.8 \%$ & $27.7 \%$ \\
\hline Saltar trepar brincar corretear & $15.5 \%$ & $11.4 \%$ & $10.3 \%$ & $25.7 \%$ & $13.4 \%$ & $30.2 \%$ \\
\hline Permanecer un rato largo quieto & $18.3 \%$ & $11.4 \%$ & $15.3 \%$ & $28.7 \%$ & $17.6 \%$ & $30.2 \%$ \\
\hline Actúa como si tuviera "un motor prendido" & $18.3 \%$ & $10.3 \%$ & $12.3 \%$ & $24.2 \%$ & $11.7 \%$ & $20.1 \%$ \\
\hline Habla demasiado en la clase o en la casa & $21.8 \%$ & $17.2 \%$ & $19.3 \%$ & $32.1 \%$ & $21 \%$ & $36.1 \%$ \\
\hline Contesta o actúa sin pensar, o escuchar & $17.2 \%$ & $13.7 \%$ & $15.8 \%$ & $23.7 \%$ & $15.9 \%$ & $29.4 \%$ \\
\hline Hacer filas o esperar turno & $16 \%$ & $9.1 \%$ & $14.3 \%$ & $20.2 \%$ & $12.6 \%$ & $23.5 \%$ \\
\hline Interrumpe las actividades y conversaciones & $18.3 \%$ & $11.4 \%$ & $15.3 \%$ & $23.2 \%$ & $11.7 \%$ & $24.3 \%$ \\
\hline \multicolumn{7}{|l|}{ HIPERACTIVIDAD } \\
\hline Terminar las tareas por si mismo & $21 \%$ & $11.4 \%$ & $19.3 \%$ & $22.7 \%$ & $21 \%$ & $26 \%$ \\
\hline Distracción con estímulos irrelevantes & $24 \%$ & $13.7 \%$ & $21.7 \%$ & $35.6 \%$ & $25.2 \%$ & $40.3 \%$ \\
\hline Mover manos y pies al estar sentado & $20.6 \%$ & $14.9 \%$ & $12.8 \%$ & $28.7 \%$ & $13.3 \%$ & $33.6 \%$ \\
\hline $\begin{array}{l}\text { Hacer ruidos, zumbidos, chiflidos y } \\
\text { resoplidos }\end{array}$ & $11.4 \%$ & $4.5 \%$ & $6.4 \%$ & $11.8 \%$ & $10 \%$ & $11.7 \%$ \\
\hline Excitabilidad, impulsividad & $10.3 \%$ & $6.8 \%$ & $6.9 \%$ & $12.3 \%$ & $10 \%$ & $16.8 \%$ \\
\hline
\end{tabular}




\section{Tabla 3}

Porcentajes de frecuencias de conductas de nivel de riesgo para los diferentes reactivos según el grupo de edad para Oposicionismo Desafiante, Agresividad y Conducta

\begin{tabular}{|c|c|c|c|c|c|c|}
\hline \multirow{3}{*}{ OD / AGRESIÓN DESAFIANTE } & \multirow{2}{*}{\multicolumn{2}{|c|}{$\begin{array}{c}\mathbf{4 - 7} \\
87(100 \%)\end{array}$}} & \multirow{2}{*}{\multicolumn{2}{|c|}{$\begin{array}{c}\mathbf{8 - 1 2} \\
202(100 \%)\end{array}$}} & \multirow{2}{*}{\multicolumn{2}{|c|}{$\begin{array}{c}13-17 \\
119(100 \%)\end{array}$}} \\
\hline & & & & & & \\
\hline & Maestros & Padres & Maestros & Padres & Maestros & Padres \\
\hline Rabietas o pataletas & $20.6 \%$ & $17.2 \%$ & $2.9 \%$ & $25.2 \%$ & $37.8 \%$ & $35.2 \%$ \\
\hline $\begin{array}{l}\text { Discusión gritos con } \\
\text { irrespeto }\end{array}$ & $9.1 \%$ & $13.7 \%$ & $7.4 \%$ & $17.8 \%$ & $12.8 \%$ & $21 \%$ \\
\hline Negarse a obedecer & $12.6 \%$ & $10.3 \%$ & $9.4 \%$ & $22.2 \%$ & $9.2 \%$ & $25.2 \%$ \\
\hline $\begin{array}{l}\text { Intencionalmente hacer } \\
\text { enojar }\end{array}$ & $10.3 \%$ & $10.3 \%$ & $9.9 \%$ & $17.8 \%$ & $8.4 \%$ & $25.2 \%$ \\
\hline Culpar a otros de errores & $10.3 \%$ & $14.9 \%$ & $10.3 \%$ & $23.2 \%$ & $8.4 \%$ & $24.3 \%$ \\
\hline Enojarse por cualquier cosa & $14.9 \%$ & $17.2 \%$ & $12.8 \%$ & $34.1 \%$ & $10 \%$ & $46.2 \%$ \\
\hline $\begin{array}{l}\text { Disgusto y resentimiento } \\
\text { frecuente }\end{array}$ & $11.4 \%$ & $14.9 \%$ & $9.9 \%$ & $31.6 \%$ & $10 \%$ & $39.4 \%$ \\
\hline Desquitarse o vengarse & $9.1 \%$ & $10.3 \%$ & $8.9 \%$ & $10.3 \%$ & $31 \%$ & $15.1 \%$ \\
\hline Buscar peleas y problemas & $8 \%$ & $5.7 \%$ & $6.4 \%$ & $9.9 \%$ & $7.5 \%$ & $10.9 \%$ \\
\hline $\begin{array}{l}\text { Dificultades para colaborar } \\
\text { o cooperar }\end{array}$ & $6.8 \%$ & $4.5 \%$ & $7.9 \%$ & $15.3 \%$ & $30.2 \%$ & $19.3 \%$ \\
\hline $\begin{array}{l}\text { Pasar de "vivo" y querer } \\
\text { sacar ventaja }\end{array}$ & $6.8 \%$ & $9.1 \%$ & $6.9 \%$ & $12.8 \%$ & $11.7 \%$ & $18.4 \%$ \\
\hline \multicolumn{7}{|l|}{ PROBLEMAS DE CONDUCTA } \\
\hline $\begin{array}{l}\text { agresividad, golpear, } \\
\text { morder, pellizcar }\end{array}$ & $1.2 \%$ & $4.5 \%$ & $4.9 \%$ & $8.4 \%$ & $21 \%$ & $9.2 \%$ \\
\hline $\begin{array}{l}\text { Dañar o destruir lo ajeno } \\
\text { con intención }\end{array}$ & $5.7 \%$ & $2.2 \%$ & $1.9 \%$ & $4.4 \%$ & $18,4 \%$ & $4.2 \%$ \\
\hline $\begin{array}{l}\text { Robar, Hurtar, estafar } 0 \\
\text { hacer trampa }\end{array}$ & $12.6 \%$ & $6.8 \%$ & $13.3 \%$ & $5.4 \%$ & $18,4 \%$ & $3.3 \%$ \\
\hline $\begin{array}{l}\text { Violación normas colegio y } \\
\text { hogar }\end{array}$ & $5.7 \%$ & $4.5 \%$ & $6.9 \%$ & $5.9 \%$ & $5.8 \%$ & $8.4 \%$ \\
\hline $\begin{array}{l}\text { Infracciones y violación de } \\
\text { derechos }\end{array}$ & $5.7 \%$ & $6.8 \%$ & $4.4 \%$ & $3.4 \%$ & $3.3 \%$ & $4.2 \%$ \\
\hline
\end{tabular}

En la tabla 3, se puede observar como sobresalen en porcentaje el ítem de rabietas y pataletas en las edades de 13 a 17 años; en el ítem de desquitarse o vengarse, para la misma edad se observa que hay una diferencia del $15.9 \%$, entre los resultados de la prueba realizada a los grupos de padres y maestros. El registro para conductas de riesgo en aspectos vinculados a la atención difiere en las apreciaciones del medio escolar y familiar, en especial para las edades de siete años en adelante. Con la misma diferencia de apreciaciones se observa un mayor registro de frecuencias de conductas de agresión desafiante que es mayor en las apreciaciones de los padres. Los problemas de conducta ofrecen un registro más alto en la apreciación de los profesores para la población adolescente. 


\section{Tabla 4}

Mediana de frecuencias absolutas y relativas de conductas en valores de riesgo para las diferentes categorías conductuales externalizantes del SNAP IV según registros de padres y maestros

\begin{tabular}{|l|c|c|c|c|c|c|}
\hline \multirow{2}{*}{} & \multicolumn{2}{|c|}{$\mathbf{4 - 7 ^ { * }}$} & \multicolumn{2}{c|}{$\mathbf{8 - 1 2 *}$} & \multicolumn{2}{c|}{13 - 17* } \\
& \multicolumn{2}{|c|}{$87(100 \%)$} & \multicolumn{2}{c|}{$202(100 \%)$} & \multicolumn{2}{c|}{119 (100\%) } \\
& Maestros & Padres & Maestros & Padres & Maestros & Padres \\
\hline Inatención & 19 & 11 & 42 & 58 & 25 & 36 \\
Ha- impulsividad & $21 \%$ & $12.6 \%$ & $20.7 \%$ & $28.7 \%$ & $21 \%$ & $30.2 \%$ \\
Inatención - & 17 & 12 & 29 & 52 & 16 & 33 \\
sobreactividad & $15.5 \%$ & $13.7 \%$ & $14.3 \%$ & $25.7 \%$ & $13.4 \%$ & $27.7 \%$ \\
TOD- agresión & 18 & 10 & 26 & 46 & 12 & 31 \\
desafiante & $20.6 \%$ & $11.4 \%$ & $12.8 \%$ & $22.7 \%$ & $10 \%$ & $26 \%$ \\
Índice Conners & 8 & 9 & 18 & 36 & 12 & 29 \\
Trastorno de & $9.1 \%$ & $10.3 \%$ & $8.9 \%$ & $17.8 \%$ & $10 \%$ & $24.3 \%$ \\
conducta & 9 & 10 & 17 & 46 & 12 & 34 \\
\hline
\end{tabular}

* Grupo por rangos de edad evaluados

La tabla 4, identifica las medianas de las frecuencias de estas conductas de riesgo observadas en cada una de las dimensiones conductuales. En general éstas no muestran mayores diferencias de registro entre padres y maestros para las edades de 4 a 7 años, pero si en el registro entre las observaciones realizadas por los padres y los registros realizados por los profesores, en los otros grupos de edad. Valores que en algunos casos se duplican o más, como es el caso de la impulsividad, hiperactividad, agresión desafiante y trastorno de conducta en los adolescentes de 13 a 17 años.

Para el análisis de la tabla 5, si se establece como patrón de comparación los criterios del DSMIV : 6/9 criterios requeridos para inatención corresponderían a una manifestación de conductas equivalentes a un $66 \%$, al igual que para hiperactividad $6 / 9$, se puede observar que desde este punto de vista, en el porcentaje de conducta equivalente estimado para la media de cada una de las categorías evaluadas, estaría en la media de normalidad la presencia de cinco de los seis criterios para un nivel de significación clínica, con una frecuencia similar en los grupos de edad. La manifestación de conductas para oposicionismo desafiante, agresión y trastorno de conducta están cercanos al $30 \%$ en todos los grupos evaluado. 


\section{Tabla 5}

Media, desviación y porcentaje de conducta observado para conductas externalizantes de acuerdo al valor de media, según los reportes de maestros y padres en los diferentes grupos de edades

\begin{tabular}{|c|c|c|c|c|c|c|c|}
\hline & \multirow[b]{2}{*}{$\begin{array}{c}\mathrm{P} \\
\text { Máxima }\end{array}$} & \multicolumn{3}{|c|}{ MAESTROS } & \multicolumn{3}{|c|}{ PADRES } \\
\hline & & Media & Ds & Conducta & Media & Ds & Conducta \\
\hline \multicolumn{8}{|l|}{$\begin{array}{c}\text { NIÑOS DE } 4 \text { A } 7 \\
\text { AÑOS }\end{array}$} \\
\hline \multirow{8}{*}{$\begin{array}{l}\text { Inatención } \\
\text { Hiperactividad } \\
\text { impulsividad } \\
\text { Inatención-Sobre } \\
\text { actividad } \\
\text { Índice Conners } \\
\text { Oposicionismo desafiante } \\
\text { Agresión desafiante } \\
\text { Trastorno de conducta } \\
\text { NIÑOS DE } 8 \text { A } 12 \\
\text { AÑOS }\end{array}$} & 27 & 12.3 & 5.7 & $46 \%$ & 12.0 & 4.8 & $45 \%$ \\
\hline & 27 & 11.7 & 6.0 & $43 \%$ & 12.0 & 4.9 & $45 \%$ \\
\hline & 15 & 6.2 & 2.9 & $42 \%$ & 6.41 & 2.4 & $42 \%$ \\
\hline & 30 & 11.9 & 4.9 & $40 \%$ & 12.8 & 4.9 & $43 \%$ \\
\hline & 33 & 8.43 & 4.2 & $35 \%$ & 10.8 & 4.2 & $32 \%$ \\
\hline & 15 & 5.11 & 2.4 & $34 \%$ & 6.15 & 2.6 & $41 \%$ \\
\hline & 15 & 4.66 & 2.2 & $31 \%$ & 4.42 & 1.2 & $30 \%$ \\
\hline & & & & & & & \\
\hline \multirow{8}{*}{$\begin{array}{l}\text { Inatención } \\
\text { Hiperactividad } \\
\text { impulsividad } \\
\text { Inatención-Sobre } \\
\text { actividad } \\
\text { Índice Conners } \\
\text { Oposicionismo desafiante } \\
\text { Agresión desafiante } \\
\text { Trastorno de conducta } \\
\text { JÓVENES DE 13 A } 17 \\
\text { AÑNOS }\end{array}$} & 27 & 11.96 & 5.4 & $44 \%$ & 13.05 & 5.1 & $48 \%$ \\
\hline & 27 & 10.8 & 5.0 & $40 \%$ & 12.8 & 5.1 & $46 \%$ \\
\hline & 15 & 5.7 & 2.4 & $38 \%$ & 6.6 & 2.4 & $46 \%$ \\
\hline & 30 & 11.1 & 4.5 & $37 \%$ & 13.2 & 4.7 & $44 \%$ \\
\hline & 33 & 8.62 & 4.05 & $26 \%$ & 10.8 & 4.2 & $32 \%$ \\
\hline & 15 & 5.1 & 2.4 & $34 \%$ & 6.1 & 2.4 & $41 \%$ \\
\hline & 15 & 4.5 & 1.72 & $30 \%$ & 4.7 & 1.7 & $31 \%$ \\
\hline & & & & & & & \\
\hline \multirow{7}{*}{$\begin{array}{l}\text { Inatención } \\
\text { HA Impulsividad } \\
\text { Inatención-Sobre } \\
\text { actividad } \\
\text { Índice Conners } \\
\text { Oposicionismo desafiante } \\
\text { Agresión desafiante } \\
\text { Trastorno de conducta }\end{array}$} & 27 & 12.0 & 5.1 & $44 \%$ & 12.07 & 4.7 & $45 \%$ \\
\hline & 27 & 11.1 & 4.8 & $41 \%$ & 11.6 & 4.6 & $43 \%$ \\
\hline & 15 & 6.1 & 2.5 & $40 \%$ & 6.3 & 2.1 & $42 \%$ \\
\hline & 30 & 11.5 & 4.4 & $38 \%$ & 12.7 & 4.5 & $42 \%$ \\
\hline & 33 & 8.7 & 3.5 & $26 \%$ & 10.5 & 4.2 & $32 \%$ \\
\hline & 15 & 5.3 & 2.2 & $35 \%$ & 5.8 & 2.3 & $39 \%$ \\
\hline & 15 & 4.5 & 1.6 & $30 \%$ & 4.6 & 1.8 & $31 \%$ \\
\hline
\end{tabular}




\section{Discusión y conclusiones:}

La frecuencia con que se presentan conductas externalizantes y la proporción de manifestaciones conductuales negativas de las mismas, pueden ofrecer alarmas para los procesos adaptativos en la población escolar y sugieren la presencia de conductas con alta probabilidad de riesgo que pueden ser intervenidas en el medio escolar por medio de una gestión razonada de la disciplina en la que se tenga en cuenta los factores de riesgo psicosociales y los aspectos del desarrollo que pueden influir en esta situación.

La infancia y la adolescencia son periodos del desarrollo que muestran que las conductas externalizadas pueden observarse con una manifestación moderada, de las cuales es esperable que tiendan a disminuir como reflejo de las luchas por los cambios del desarrollo (Campbell, 1995). Estas conductas aun así, pueden permanecer o incrementarse inapropiadamente. En el caso de la población evaluada se observa una estabilidad en la frecuencia de manifestaciones de estos comportamientos en los diferentes grupos de edad, resultados que resaltan la importancia de identificar los factores de riesgo para evitar que estas expresiones pasen de ser un proceso normal de ajuste social a un problema de conducta crónico.

Estos comportamientos latentes que en un momento del desarrollo pueden observarse ocasionalmente, cobran especial interés sobretodo cuando están inmersos en situaciones familiares, escolares y sociales mediatizadas por la intolerancia y la violencia (Wicks \& Israel, 2000). Ésta es una de las razones que justifica evaluar desde una perspectiva descriptiva las conductas disruptivas que están presentes en nuestra población en el ciclo de la niñezadolescencia y la relación que éstas conductas tienen con otras dimensiones del desarrollo que le son afines, como lo es el razonamiento moral, la autorregulación y el ajuste social.

Verhulst y Van der Ende (1991) (como cita en Montiel \& Peña, 2001) comunicaron que el acuerdo y consistencia entre las observaciones de padres, profesores y niños fue mayor para las conductas externas (negativismo, agresión, hiperactividad) que para las conductas internas (ansiedad, depresión, timidez).Con relación a estos resultados se observa un mayor registro de los profesores para las conductas externalizantes en las edades de 4 a 7 años, pero en las edades mayores no hay la consistencia esperada entre las observaciones de los padres y maestros, apreciaciones que en general son más altas en los padres de los niños-adolescentes entre los 8 y 17 años de edad.

En consonancia con los resultados obtenidos, un metanálisis acerca de las relaciones entre las descripciones de diferentes informantes, Achenbach et al (1995) (como cita en Montiel \& Peña, 2001), encontraron que existe un acuerdo mayor entre las evaluaciones de informantes similares (p. ej., ambos padres o profesores y profesionales de la salud mental), que entre las evaluaciones 
de diferentes tipos de informantes (padres/profesores); estas conclusiones se han corroborado en otros estudios (Stranger \& Lewis, 1993; Jensen, Rubio-Stipec, Canino, Bird, Dulcan, Schwab, et al., 1999) (como cita en Montiel \& Peña, 2001).

Las diferencias en la información provista por cada una de las fuentes no necesariamente implica que una sea más válida que la otra, sino que puede interpretarse también como un reflejo de las diferencias conductuales ocasionadas por los diversos contextos o a las interacciones diferenciales entre el niño y quienes informan (Steele, Forehand \& Devine, 1996). A pesar de los desacuerdos entre los diferentes informantes, la perspectiva de cada uno de ellos puede contribuir válidamente a una evaluación completa de las necesidades del niño (Achenbach \& Edelbrock, 1984).

Steele, Forehand y Devine (1996) (como cita en Montiel \& Peña, 2001), proponen que dichas diferencias no se deben interpretar como errores de la validez de las observaciones o de los instrumentos utilizados, sino más bien como una función de las diferencias de interrelaciones entre el informante y el niño.

Los programas de prevención orientados a la población normal deben tener en consideración la autorregulación cognitiva y conductual, las pautas de crianza, estilos de vida, normas, estructura familiar, oportunidades socio-culturales, entre otros factores, ya que son importantes e influyentes en la determinación del ajuste o no ajuste social y en especial para intervenir oportunamente las conductas disruptivas y de comportamiento perturbador.

Un ambiente organizado, con reglas claras, aprendizajes sistemáticos y oportunidades de autoreflexión permanente son positivos para que el niño y/o adolescente pueda autorregularse para las demandas escolares y familiares e interactuar positivamente con los demás. De esta manera realizar un acercamiento hacia la resolución activa de problemas, la toma de decisiones y las habilidades de comunicación contribuyen a este propósito.

El acompañamiento adecuado para el desarrollo cognitivo y cultural unido a los aspectos del desarrollo moral son importantes para favorecer la conducta prosocial pero probablemente no son suficientes y explicativos de la naturaleza y manifestaciones de las conductas disruptivas externalizantes, las cuales requieren una comprensión clara de los factores de riesgo que las producen, las diferencias individuales y las prácticas que se generan desde los agentes externos.

El desarrollo de la autorregulación cognitiva es proactiva para el logro de habilidades de reflexión, autonomía, comunicación, autocontrol y de conductas prosociales. Esta se expresa en 
una autonomía conductual que acompañada de un sistema de normas claras, con sanciones definidas y alcanzables,

constituyen un marco preventivo para las conductas disruptivas y de comportamiento perturbador.

No puede alcanzarse la autorregulación sin la correspondencia de ésta con la regulación grupal y social. Para esto, es importante tener en cuenta la familia, el vínculo con ella, el nivel de inserción social y las relaciones interpersonales. 


\section{Referencias Bibliográficas}

Achenbach, T. \& Rescorla, L. (2001). Manual for the ASEBA School:

Age Forms \& Profiles. Child Behavior Checklist for Ages 6-18, Teacher's Report from, Youth self-report an Integrated System of Multi-informant Assessment. Burlington: University of Vermont.

Achenbach, T.M., Howell, C.T., McConaughy, S.H. \& Stanger,

C.(1995). Six-year predictors of problems in a national sample of children and youth: I. Crossinformant syndromes. Journal American Academy Child Adolescent Psychiatry, 34, $336-47$

Achenbach, T.M. \& Edelbrock, C.S. (1984). Psychopathology of childhood. Ann Rev Psychology, 35, 227-56

American Psychiatric Association. (2002). Diagnostic and statistical manual of mental disorder (DSM IV-TR) (4a ed). Washington DC: American Psychiatric Association

Anderson, S.W., Bechara, A., Damasio, H., Tranel, D., \& Damasio, A.R. (1999). Impairment of social and moral behavior related to early damage in human prefrontal cortex. Nature Neuroscience, 2(1 1), 1032-103

Brown, T.E. (2003). Trastornos por déficit de atención y comorbilidades en niños, adolescentes y adultos. Barcelona: Masson

Campbell, S.B. (1995). Behavior problems in preschool children. A review of recent research. Journal of child psychology and psyquiatry, 36, 11 3-149

Cano, I.C. \& Castillo, N.M. (2002). Factores prenatales asociados con el trastorno disocial de la conducta en adolescentes infractores de la ciudad de Medellín. Universidad de San Buenaventura, Medellín, Colombia

Colby, A. \& Kohlberg, I. (1987) The Measurement of Moral Judgment (Vol. 1-2). New York: Cambridge University Press 
Conner, C.k.(1979). Conner Parents Rating Scale (Hyperkinesis index). Chicago, II: Abbott Laboratories

Jennings, K.D., Sandberg, I., Kelley, S.A., Valdes, L., Yaggi, K., Abrew, A. et al. (2008). Understanding of self and maternal warmth predict later selfregulation in toddlers. International Journal of Behavioral Development, 32, 108

Kerr, D., López, N., Olson, S. \& Sameroff, A. (2004). Parental Discipline and Externalizing Behavior Problems in Early Childhood: The Roles of Moral Regulation and Child Gender. Journal of Abnormal Child Psychology, Vol. 32

Kamphaus, R \& Frick, P (1992). Evaluación Clínica de la Personalidad y la Conducta del Niño y el Adolescente. México. Manual Moderno.

Millich, R. \& Loney, J. (1985). Structured observation of academic and play setting. lowa: University of lowa

Mischel, W. \& Rodriguez, M. L. (1993). Psychological distance in self-imposed delay of gratification New York: Guilford.

Montiel Nava, C. \& Peña, J.A. (2001). Discrepancia entre padres y profesores en la evaluación de problemas de conducta y académicos en niños y adolescentes. Revista de Neurología, 32, 506-511

Reynold, C.R. \& Kamphaus, R.W. (1992). BASC. Behavior Assessment System for Children. AGS American Guidance Service, Inc

Sattler, J. (2001). Evaluación Infantil: Aplicaciones cognitivas. California L.A: Manual moderno

Steele, R., Forehand, R. \& Devine, D. (1996). Adolescent social and cognitive competence: crossinformant and intraindividual consistency across three years. Journal Clinical Child Psychology, 25, 605

Stanger, C. \& Lewis, M. (1993). Agreement among parents, teachers, and children on internalizing and externalizing behavior problems. Journal Clinical Child Psychology, 22, 107-15 
Swanson, J.M., Sergeant, J.A., Taylor, E., Sonuga-Barke, E.J.S., Jensen, P. \& Cantwell, D.P. (1998). Attention-deficit hyperactivity disorder and hyperkinetic disorder. The Lancet, 35 1, 429-433

Swanson, J.M., Wigal, S.B., Udrea, D., Lerner, M., Agler D., Mylnn, D. et al.(1998). Evaluation of individual subjects in the analog classroom setting, I: examples of graphical and statistical procedures for within-subject ranking of responses to different delivery patterns of methylphenidate. Psychopharmacol Bull, 34, 825-832

Toro, G. \& Yepes, R. (2004). Fundamentos de medicina. Psiquiatría ( $4^{\mathrm{a}}$ ed). Medellín: CIB

Verhulst, FC. \& Van der Ende, J. (1991). Assessment of child psychopathology: relationships between different methods, different informants and clinical judgment of severity. Acta Psychiatry Scand, 84, 155-9

Wicks Nelson, R. \& Allen, I. (1997/2000). Psicopatología del niño y del adolescente ( ${ }^{\text {a }}$ Reimpresión). España: Prentice Hall 